\title{
Diagnosis
}

\section{Insufficient evidence on benefits of early detection and treatment of dementia}

\author{
Boustani M, Peterson B, Hanson L et al. Screening for dementia in primary care: a summary of the evidence for the U.S. \\ preventative services task force. Ann Int Med 2003;138:927-937.
}

\section{QUESTION: What are the benefits and harms of screening for, and treating dementia?}

Design

Systematic review with narrative synthesis.

\section{Data sources}

The authors searched Medline, PsychINFO, EMBASE and the Cochrane library. Reference lists were hand searched and authors contacted.

\section{Study selection}

Studies had to be in people aged 60 years or more, and published in English between January 1994 and September 2002. Eligible studies for evidence on screening, prevalence of under diagnosed dementia and accuracy of screening tests included systematic reviews, randomised controlled trials, cross-sectional prevalence studies and prospective cohort studies. Evidence on treatment efficacy was assessed from systematic reviews and randomised controlled trials.

\section{Data extraction}

2 reviewers extracted data on prevalence of under diagnosed dementia, accuracy of screening tests, and efficacy of treatments, using a standardised data extraction form.

\section{Main results}

Screening: screening for dementia has not been assessed in any randomised controlled trials. 4 crosssectional studies in primary care populations reported undiagnosed dementia in $1.8-12 \%$ of the study population. 1 systematic review and 9 additional studies examined the diagnostic accuracy of brief screening instruments. Assuming a 10\% prevalence of undiagnosed dementia, the positive predictive value of 5 of the more accurate tests (mini mental state, functional activities questionnaire, short test of mental status, Blessed information memory concentration, Blessed orientation memory concentration) was $40-50 \%$, with a negative predictive value of $95 \%$ or more. The review found no studies examining harms of screening.

Treatment: the review found no studies examining treatment as a result of early detection of dementia. 4 systematic reviews and 5 additional randomised controlled trials showed that cholinesterase inhibitors slow the decline in cognitive scores, but the clinical relevance in terms of functional ability was not clear. Studies found that ginkgo biloba, selegiline, antidepressants, vitamin E and oestrogen did not slow cognitive decline.

\section{Conclusions}

There is insufficient evidence on this topic. The benefits and harms of screening for dementia are unknown.

\section{COMMENTARY}

This article calls to mind two conversations I have had recently. A competent colleague in general practice asked me "what is the point in trying to detect dementia early in the course of the illness?" There are several issues behind this question (how, why, when and in whom?) and the article by Boustani et al neatly addresses these. Unfortunately, this article highlights the paucity of evidence for the validity of screening instruments, the efficacy of early interventions and the impact on patients of being given the diagnosis. So perhaps we should not be too worried that the article suggests that many individuals with dementia go undiagnosed. In short, my GP colleague would have found plenty of ammunition to support her argument.

Of course clinicians working in the field of dementia and their patients and carers will seize on any evidence that is available to support current practices of diagnosis and intervention, while bleating about the reductionism inherent in the evidence-based culture of health care delivery where evidence is lacking. Evidence for dementia care practices is accumulating, but currently resembles a house of cards, built on the twin weak foundations of a focus on Alzheimer's disease (there are very few studies on the other $50 \%$ of dementias) and difficulties in measuring meaningful outcomes.

This brings me to the second conversation. I had given a talk advocating caution in over-interpreting results from cholinesterase inhibitor trials. Afterwards, a carer of someone with dementia told me that since his wife started cholinesterase inhibitors she now recognises him as her husband again and is much happier. Although the impact of this one small change would be lost in the noise of a clinical trial, his life had turned around as a result.

James P Warner, MD MRCPsych Senior Lecturer in Old Age Psychiatry Imperial College London, UK

Boulevard, RG6

Indianapolis, IN 46202-2872. 